\title{
Alternative to traditional noninvasive ventilation using a modified snorkel mask in a patient with SARS-COV2: A case report
}

\author{
Raul Montalvo ${ }^{1}$, Eyner Castro ${ }^{2}$, Alvaro Chavez ${ }^{3}$
}

\begin{abstract}
R Montalvo, E Castro, A Chavez. Alternative to traditional noninvasive ventilation using a modified snorkel mask in a patient with SARS-COV2: A case report. Can J Respir Ther 2021;57:18-21. doi: 10.29390/cjrt-2020-039.

Introduction: The lack of mechanical ventilators for patients with COVID-19 has necessitated the use of other noninvasive ventilation (NIV) systems. One of these NIV systems is the use of an adapted snorkel mask with inspiration valves and pressure valve (PEEP).

Case and outcomes: A 48-year-old man with no previous history of lung disease was admitted to the emergency room with a diagnosis of acute respiratory failure due to SARS-COV2. The patient did not improve saturation with the use of the reservoir mask. Oxygenation was started using an adapted snorkel mask with a PEEP valve with an alveolar recruitment function and double oxygen flow. The patient presented clinical and radiological improvement after 2 days of use and was discharged 16 days later.

Discussion: The use of a snorkel mask is an important, viable, and simpler NIV modality for the management of patients with COVID-19 with respiratory failure who fail to use a reservoir mask, and it can be an alternative before the use of a mechanical ventilator.

Conclusion: The use of the adapted snorkel mask with Charlotte valve and PEEP is a feasible alternative for the treatment of patients with COVID-19.
\end{abstract}

Key Words: noninvasive ventilation; mechanic ventilation; COVID-19; SARS-COV-2

\section{INTRODUCTION}

Since the beginning of the pandemic, the mechanical ventilation (MV) of patients with acute respiratory failure due to COVID-19 has caused more than one headache for doctors due to the high demand for mechanical ventilators and the absence of beds in the intensive care unit. In this sense, the use of noninvasive ventilation systems (NIV) has evolved rapidly.

The World Health Organization recommends NIV for patients with hypoxemic respiratory failure due to COVID-19 in the absence of available mechanical ventilators, with strict follow-up by specialized personnel to perform rapid orotracheal intubation if necessary and with extreme caution, because of a potential risk of aerolization $[1,2]$.

Recently, an adapted snorkel mask has been used as protective equipment for medical personnel [3]. Given the shortage of mechanical ventilators, a snorkel mask adapted with inspiration valves and the pressure valve (PEEP) (range: $5-20 \mathrm{~cm} \mathrm{H}_{2} \mathrm{O}$ ) to the expiratory line can also provide positive pressure, becoming an alternative to NIV for patients [4]. This mask has the advantage of being washable, disinfectable, and reusable.

This case report presents the gasometric and radiological evolution of a patient with Acute Respiratory Distress Syndrome (ARDS) due to COVID-19 who presented clinical improvement after the use of the snorkel mask.

\section{CASE REPORT}

Institutional review board approval for this project was formally waived by the institution since the sample size was less than 2 patients.

This report presents the case of a 48-year-old male patient with no previous pathologies. He went to the emergency room after 10 days of headache, fever, general malaise, dry cough and, for the 3 days prior, dyspnea with small efforts. On examination, the patient's measurements were as follows: weight $84 \mathrm{~kg}$, body mass index $31 \mathrm{~kg} / \mathrm{m}^{2}$, heart rate $103 x^{\prime}$, respiratory rate 29x', blood pressure $100 / 60 \mathrm{mmHg}$, saturation $72 \%$, and temperature $37.4^{\circ} \mathrm{C}$. On pulmonary examination, crepitus was evidenced in the lower two-thirds of both lung fields and scant expiratory wheezing located in the middle third of the right hemithorax.

Oxygen supply was started with a binasal cannula at $5 \mathrm{~L}$ per minute. When there was no improvement, the patient was changed to a $15-\mathrm{L}$ reservoir mask, improving saturation to $86 \%$. Laboratory tests showed positive IgM and IgG rapid test for COVID-19, glycosylated hemoglobin 6.2, lactic dehydrogenase $987 \mathrm{u} / \mathrm{L}$, and C-reactive protein 96 . The rest of the tests are shown in Table 1.

With these results, the patient was placed in the prone position and began treatment with dexamethasone $20 \mathrm{mg}$ intravenously once a day for 3 days. This was continued with dexamethasone $6 \mathrm{mg}$ once a day for 7 days, enoxaparin $60 \mathrm{mg}$ subcutaneous injection once a day; ranitidine $50 \mathrm{mg}$ intravenously 2 times a day, sodium chloride $9 / 1000 \mathrm{~mL}$, paracetamol $500 \mathrm{mg}$ orally 3 times a day, acetylcysteine $300 \mathrm{mg}$ intravenously 3 times a day, and salmeterol/fluticasone.

On the second day of evolution, the patient presented a clinical worsening with a decrease in oxygen saturation, a decrease in blood gas parameters (Table 1), and intolerance to pronation. The intensive care physician reported the absence of MV, so it was decided to use the snorkel mask with the prior consent of the patient and their family members. The snorkel mask was connected to a double oxygen port of $15 \mathrm{~L}$, each directed to the venturi system and to the reservoir mask that was attached to the inspiratory valve (Figure 1). The snorkel mask was connected to the PEEP valve. This valve was adjusted to $0 \mathrm{~cm} \mathrm{H}_{2} \mathrm{O}$, and after 3 min of

\footnotetext{
${ }^{1}$ Faculty of Human Medicine, National University of the Center of Peru, Huancayo, Peru;

${ }^{2}$ Department of Medicine, Hospital Daniel Alcides Carrión, Huancayo, Peru;

${ }^{3}$ Department of Medicine, Ortega Clinic, Huancayo, Peru

Correspondence: Raul Montalvo, MD, Avenida Mariscal Castilla 3909, Huancayo 12006, Peru, Tel. (064) 481062, E-mail: motivo3@gmail.com
}

Published online at https://www.cjrt.ca on 09 February 2021 
TABLE 1

Gasometric characteristics during hospital stay.

\begin{tabular}{|c|c|c|c|c|c|c|c|c|}
\hline Date & $31 / 7 / 20$ & $1 / 8 / 20$ & 2/8/20 7H. & $2 / 8 / 2016 \mathrm{H}$ & $3 / 8 / 20$ & $04 / 8 / 20$ & $06 / 8 / 20$ & $9 / 8 / 20$ \\
\hline Hospitalization day & 0 & 1 & 2 & 2.5 & 3 & 4 & 5 & 9 \\
\hline $\mathrm{PCO}_{2}$ & 38.7 & 37.2 & 41.2 & 37.4 & 39.8 & 36.2 & 39.7 & 37.4 \\
\hline $\mathrm{PO}_{2}$ & 45 & 54 & 49 & 54 & 61 & 51 & 52 & 55 \\
\hline $\mathrm{SO} 2$ & 83 & 89 & 85 & 90 & 91 & 89 & 89 & 91 \\
\hline $\mathrm{Pa} / \mathrm{FIO}_{2}$ & 56.3 & 67.5 & 61.3 & 67.5 & 76.3 & 85 & 104 & 196.4 \\
\hline $\mathrm{HCO}_{3}$ & 27 & 24.7 & 27 & 24.8 & 24.9 & 25.1 & 29 & 25.7 \\
\hline $\mathrm{PH}$ & 7.45 & 7.43 & 7.42 & 7.43 & 7.44 & 7.45 & 7.47 & 7.45 \\
\hline $\mathrm{FiO}_{2}$ & $80 \%$ & $80 \%$ & $80 \%$ & $80 \%$ & $80 \%$ & $60 \%$ & $50 \%$ & $28 \%$ \\
\hline Device used & RM 15L & RM 15L & RM 15L & $\begin{array}{c}\text { First day of } \\
\text { snorkel-casmon }\end{array}$ & $\begin{array}{l}\text { Second day of } \\
\text { snorkel-casmon }\end{array}$ & RM 10L & $\begin{array}{c}\text { VENTURI } 15 \mathrm{~L} . \\
50 \% \mathrm{FIO} 2\end{array}$ & $\mathrm{BC} 2 \mathrm{~L}$ \\
\hline
\end{tabular}

Reference values: PCO2 (35-45), PO2 (80-105 MMhG), SO2 (95\%-98\%), Na (138-146 mMOL / L), K (3.5-4.9), HCO3 (22-26). PH (7.35-7.45).

RM, Reservoir mask; BC, Binasal Cannula.

\section{FIGURE 1}

Assembled model of the snorkel mask for use as noninvasive ventilation in the patient.

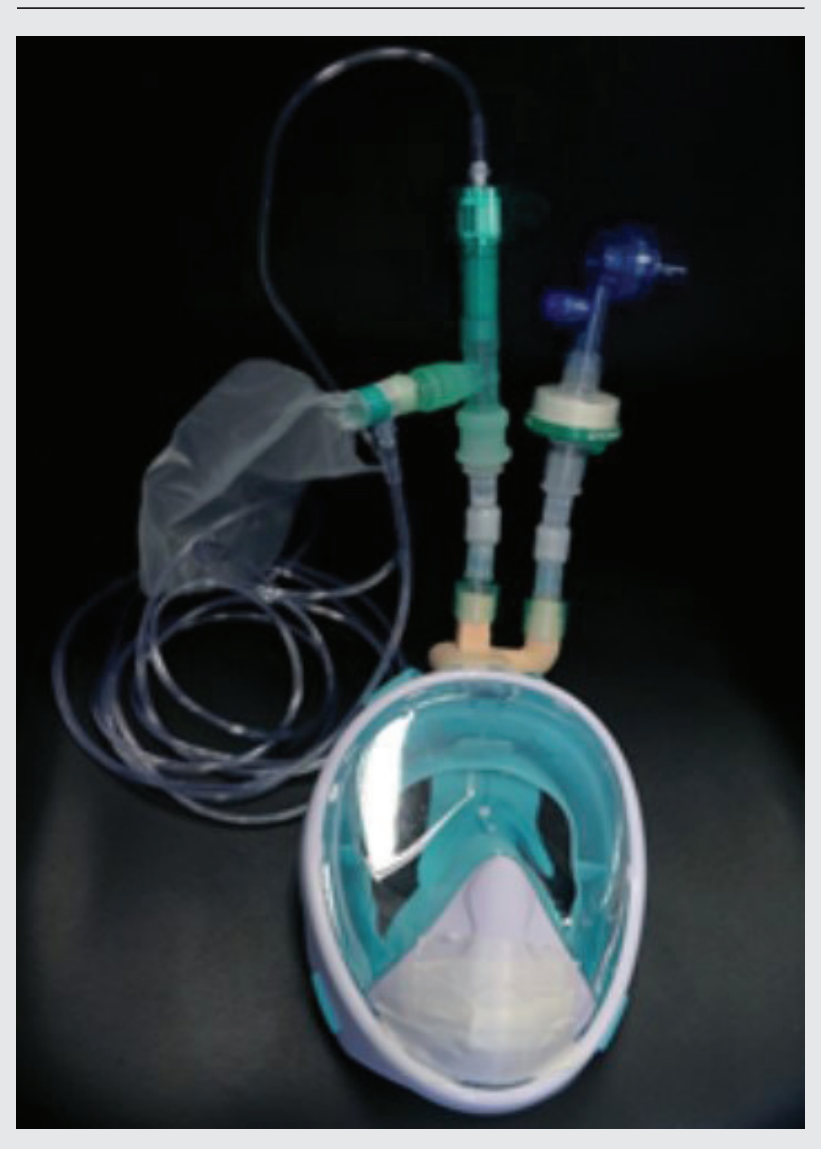

operation under medical supervision to monitor fit and avoid air leakage, an increase in saturation to $93 \%$ was evidenced. The patient remained in this state for $15 \mathrm{~min}$, then switched to a prone position. The saturation immediately decreased to $90 \%$; after 10 min the saturation increased to $93 \%$. The PEEP pressure was increased progressively every minute from 0 to 2.5 then to $5,7.5,10$, and $12 \mathrm{~cm} \mathrm{H}_{2} \mathrm{O}$, the saturation values were kept between $91 \%$ and $93 \%$. The patient remained in the prone position for $4 \mathrm{~h}$ with PEEP $12 \mathrm{~cm} \mathrm{H}_{2} \mathrm{O}$ until lunch time; this maneuver was repeated twice a day for 2 consecutive days after breakfast and after lunch. The rest of the time he remained with a reservoir mask at $15 \mathrm{~L}$ per minute, without a snorkel mask and in the prone, semi-sitting and lateral position according to tolerance. The patient remained in bed the entire time and was provided with a container for urine and feces to prevent decompensation.

On the fourth day of hospitalization, the patient did not agree to put on the mask because he reported that he felt better with the reservoir bag, so the snorkel mask was not put back on. Likewise, the patient presented progressive clinical, laboratory, and radiographic improvement (Figure 2), with evidence of improvement in lung function.

The patient remained hospitalized for 16 days. He was discharged from the hospital without oxygen requirement, with $90 \%$ saturation, continuous with acetyl cysteine $600 \mathrm{mg}$ orally 3 times a day, ranitidine $300 \mathrm{mg}$ once a day, and ibuprofen $400 \mathrm{mg}$ orally 2 times a day due to the presence of chest pain intensity reported as $3 / 10$.

\section{DISCUSSION}

With the presentation of this clinical case, it is shown that given the need for MV, other NIV systems can be used, such as the use of a snorkel mask, with satisfactory results. In a follow-up study of COVID-19 patients who required MV and underwent NIV, 77\% were not intubated, with a mortality rate of $10 \%$ [5].

Based on these results, NIV is feasible with a considerable success rate and helps prevent MV in COVID-19 patients. Increasing the use of NIV either to decrease complications related to MV and sedation or to maximize the availability of mechanical ventilators.

At the early start of the epidemic, experts recommended early intubation of COVID-19 patients to avoid self-inflicted lung injury (p-SILI) and a large tidal volume that can worsen ARDS [6]; however, these complications do not justify the routine use of MV and its prolonged use causes poor results. On the other hand, the true impact of MV is difficult to measure and it is necessary to evaluate the real need for MV and the delay in MV.

A study in Atlanta published the use of high flow nasal oxygenation as noninvasive respiratory support [7]. This study shows that delayed intubation did not interfere with mortality $(<40 \%)$ in patients with ARDS related to COVID-19, advocating for the use of NIV in COVIDARDS patients.

However, some patients, for various reasons, cannot tolerate the application of traditional noninvasive mask ventilation [8]. These factors create the need for the use of other alternatives, such as the use of the modified snorkel mask to avoid aerolization and improve oxygen flow delivery. Unlike other NIV systems that managed to reduce intubation rates [9], this snorkel system offers a double inspiratory circuit with a humidifier filter (shown in Figure 1), and an antiviral filter was connected to the exhalation circuit. The well-fitting mask with a seal on the face prevents air leakage and allows the maintenance of positive pressure in the mask.

Although the effectiveness of the snorkel mask has not been widely validated in patients with COVID-19, this case report shows that its use is feasible, preventing the patient from entering intubation.

Among the possible dangers and limitations of the use of the snorkel mask, we find that the patient breathes the exhaled air again, which 


\section{FIGURE 2}

Progression of chest images in the patient with noninvasive ventilation therapy with a snorkel mask. (A) Upon admission, with oxygen through a reservoir mask. (B) Second day with the Snorkel system. (C) Day 7 of hospitalization. (D) Day 10 of patient hospitalization.

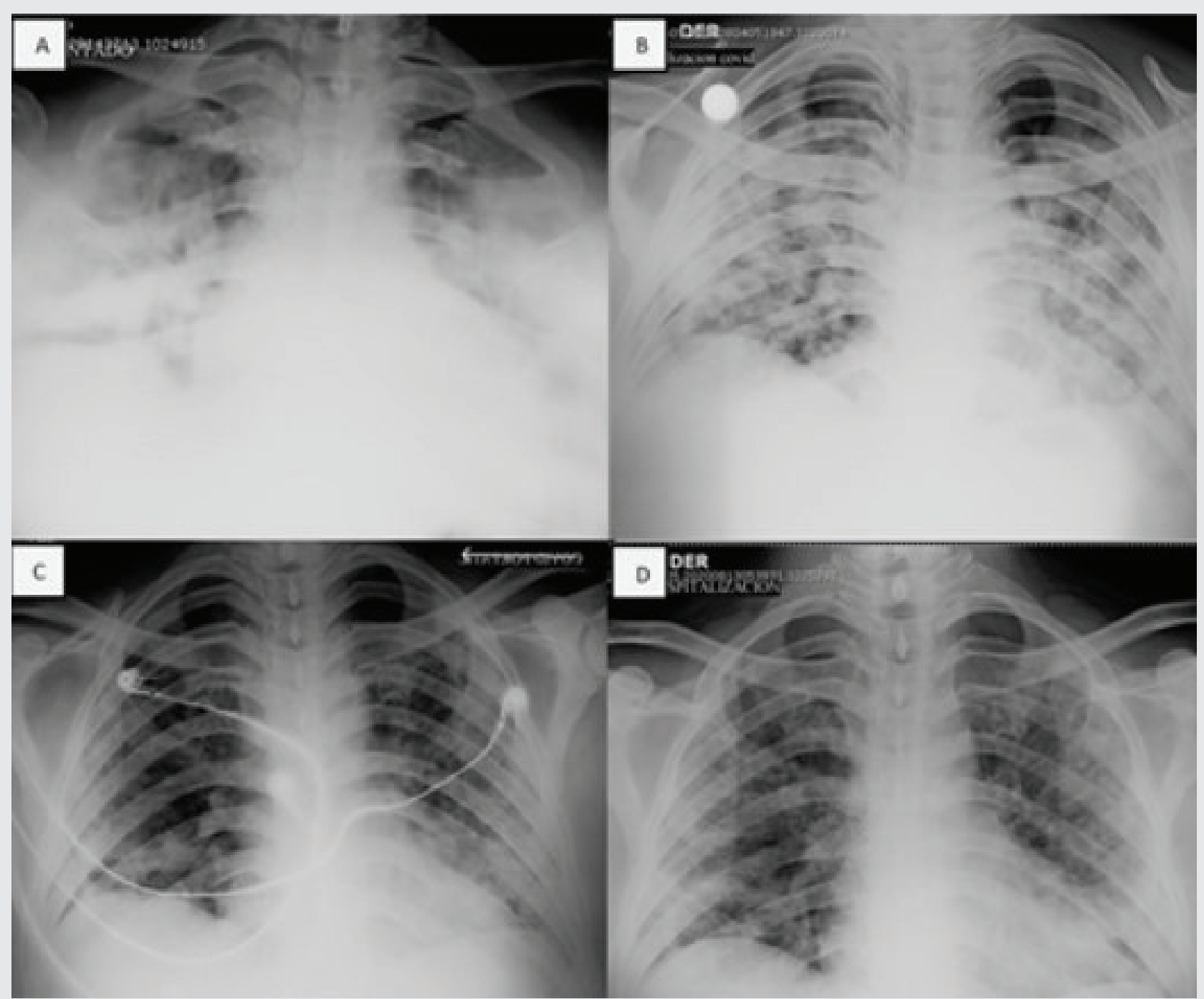

produces hypercapnia. To avoid this, flow rates (typically at least $50 \mathrm{~L}$ per minute) are needed to maintain adequate inspiratory positive airway pressure and expiratory positive airway pressure [10-12].

Another possible concern with mask use is ocular barotrauma due to positive pressure ventilation; however, no such complications were reported in a large study evaluating this interface [9]. Our patient's experience with the frequent application of artificial tears can help mitigate this risk. Mask use is not recommended for patients who experience significant hypercapnia, because they accumulate $\mathrm{CO}_{2}$ in the mask (maximum recorded: $25 \mathrm{mmHg}$ ) compared with the CPAP mask $(5 \mathrm{mmHg})$ [2]; however, in this case there was no evidence of an increase in $\mathrm{PCO}_{2}$, similar to that evidenced in others where volunteers are subjected to exercises and then exposed to a snorkel mask.

\section{CONCLUSION}

The snorkel mask presents a viable and simpler alternative for COVID-19 patients who do not respond to a reservoir mask before entering a mechanical ventilator. Recruitment maneuvers consistent with a progressive increase in PEEP pressure levels up to $12 \mathrm{~cm} \mathrm{H}_{2} \mathrm{O}$, careful evaluation of oxygen saturation, minimizing the risk of oxygen leaks, and placing the patient in the prone position using the snorkel mask should be considered to improve the answer. However, it is suggested that a randomized trial be conducted to analyze the efficacy of the use of the snorkel mask in patients with SARS-COV-2 infection who do not respond to the reservoir mask and evaluate the increase in $\mathrm{PCO}_{2}$.

\section{DISCLOSURES}

\section{Contributors}

All authors contributed to the conception or design of the work and the acquisition, analysis, or interpretation of the data. All authors were involved in drafting and commenting on the paper and have approved the final version. 


\section{Funding}

This study did not receive any specific grant from funding agencies in the public, commercial, or not-for-profit sectors.

\section{Competing interests}

All authors have completed the ICMJE uniform disclosure form at www. icmje.org/coi_disclosure.pdf and declare no financial relationships with any organizations that might have an interest in the submitted work in the previous 3 years or no other relationships or activities that could appear to have influenced the submitted work.

\section{REFERENCES}

1. World Health Organization. Clinical management of severe acute respiratory infection when novel coronavirus ( $\mathrm{nCoV}$ ) infection is suspected. 2020. Interim Guidance. March 13. Geneva: WHO; 2020. Available at: https:// www.who.int/publications/i/item/clinical-management-of-covid-19.

2. Montalvo R, Ochoa S, Quispe-Pari J, De La Cruz J, Rosales J, Gomez A. Evaluation of the economic impact for the management of patients confirmed by COVID-19 infection in Peru. Pharmacoeconomics 5(1):2020. doi: 10.37421/pe.2020.5.124.

3. Landry SA, Mann DL, Djumas L, et al. Laboratory performance of oronasal CPAP and adapted snorkel masks to entrain oxygen and CPAP. Respirology 2020. doi: 10.1111/resp.13922.

4. Rivory J, Beaugendre E, Yvon C, et al. Why not use the Easybreath snorkeling mask to prevent COVID-19 transmission during endoscopy procedures when FFP2 are lacking? Endoscopy 2020;52(8):E306-7. doi: $10.1055 / \mathrm{a}-1178-9125$.
5. Mukhtar A, Lotfy A, Hasanin A, El-Hefnawy I, El Adawy A. Outcome of non-invasive ventilation in COVID-19 critically ill patients: a retrospective observational study. Anaesth Crit Care Pain Med. doi: 10.1016/j. accpm.2020.07.012.

6. Gattinoni L, Coppola S, Cressoni M, Busana M, Rossi S, Chiumello D. Covid-19 does not lead to a "typical" acute respiratory distress syndrome. Am J Respir Crit Care Med 2020. https://www.atsjournals.org/doi/ 10.1164/rccm.202003-0817LE

7. Hernandez-Romieu AC, Adelman MW, Hockstein MA, et al. Timing of intubation and mortality among critically ill coronavirus disease 2019 patients. Crit Care Med. doi: 10.1097/CCM.0000000000004457.

8. Schwartz AR, Kacmarek RM, Hess DR. Factors affecting oxygen delivery with bi-level positive airway pressure. Respir Care 2004:49(3):270-5. Available at: http://rc.rcjournal.com/content/49/3/270.short

9. Patel BK, et al. Effect of noninvasive ventilation delivered by helmet vs face mask on the rate of endotracheal intubation in patients with acute respiratory distress syndrome: a randomized clinical trial. JAMA 2016;315(22):2435-41. doi: 10.1001/jama.2016.6338.

10. Rali AS, Howard C, Miller R, et al. Helmet CPAP revisited in COVID-19 pneumonia: a case series. Can J Respir Ther 2020;56:32-4. doi: 10.29390/ cjrt-2020-019.

11. James A, Verdonk F, Bougle A, Constantin JM. Non-invasive ventilation for acute respiratory failure (in COVID-19 patients): the non-ending story? Anaesth Crit Care Pain Med 2020. doi: 10.1016/j.accpm. 2020.08.004.

12. Reis JF, Millet GP, Malatesta D, et al. Are oxygen uptake kinetics modified when using a respiratory snorkel? Int J Sports Physiol Perform 2010;5(3):292-300. doi: 10.1123/ijspp.5.3.292. 